\title{
CARACTERIZAÇÃO DAS BOLHAS NA PANELA DE ACIARIA POR MODELAMENTO FÍSICO*
}

\author{
Pedro Henrique da Rocha Silva ${ }^{1}$ \\ Daniel Andrade de Jesus ${ }^{2}$ \\ Isabella Grama Camargo 3 \\ Rafael Silva Alves ${ }^{4}$ \\ Roberto Parreiras Tavares ${ }^{57}$
}

\section{Resumo}

Os tratamentos de borbulhamento de gás nas panelas são parte essencial para melhoria da cinética de todas as reações, diminuição de zonas mortas e remoção de inclusões. O presente trabalho teve o objetivo de caracterizar as bolhas da pluma na panela usando fotos tiradas por uma câmera de alta velocidade e assim avaliar seu diâmetro e sua área superficial. Foram utilizados três plugues porosos situados no fundo da panela, com áreas de injeção diferentes e testadas diferentes vazões de injeção. A partir dos valores encontrados, foram feitas considerações sobre os processos de refino dentro da panela.

Palavras-chave: Aciaria, Panela; Injeção de gás; Modelamento Físico; Bolhas; Área Superficial; Metalurgia Secundária.

\section{BUBBLE CHARACTERIZATION AT THE STEELMAKING LADLE BY PHYSICAL MODELING}

\section{Abstract}

Rinsing treatments are essential in secondary refining of steels. They can improve the kinetics of the reactions, reduce dead zones and promote inclusion removal. In the present work, bubbles generated during gas injection using porous plugs were characterized by means of pictures taken by a high speed camera in a physical model of a steelmaking ladle. Bubble diameters and their surface areas at different heights in the plume were evaluated. Porous plugs with different injection areas and different gas flow rates were adopted. The possible effects of the different gas injection configurations on the performance of the treatment were analyzed..

Keywords: Steelmaking; Ladle; Gas injection; Physical modeling; Bubbles; Surface Area; Secondary Steelmaking.

\footnotetext{
Engenheiro Metalurgista, Mestrando - PPGEM - UFMG, Belo Horizonte, Minas gerais, Brasil;

Graduando em Engenharia Metalúrgica, UFMG, Belo Horizonte, Minas Gerais, Brasil;

Graduando em Engenharia Metalúrgica, UFMG, Belo Horizonte, Minas Gerais, Brasil;

Graduando em Engenharia Metalúrgica, UFMG, Belo Horizonte, Minas Gerais, Brasil;

Engenheiro Metalurgista, Doutor, Professor em Engenharia Metalúrgica e de Materiais- UFMG,

Belo Horizonte, Minas Gerais, Brasil..
} 


\section{INTRODUÇÃO}

O refino secundário é a última oportunidade de modificar de forma significativa a composição química de um aço antes do seu lingotamento [1]. Por isso a panela de aciaria tem um importante papel na produção siderúrgica, uma vez que vários tratamentos são aplicados nessa etapa.

Normalmente o banho de metal na panela é agitado por borbulhamento de gás, em geral argônio ou nitrogênio, através de plugue poroso ou ventaneira posicionados no fundo da panela ou por meio de uma lança revestida de refratário introduzida no banho [2]. A injeção de gás no aço líquido pode trazer benefícios, como a homogeneização térmica e química, flotação das inclusões, melhoria das taxas de reações de refino e aceleração da dissolução de elementos de ligas $[3,4]$.

Apesar de todos os benefícios que o borbulhamento de gás pode trazer ao banho, seu uso inapropriado pode fazer com que haja abertura da camada de escória e assim deixar o aço vulnerável à atmosfera, favorecendo assim a absorção de nitrogênio e a reoxidação do aço. Outro malefício causado pelo uso inadequado de gás é o entranhamento de escoria no aço [5], que pode ter efeitos negativos para o produto final.

O uso do gás pode resultar em diminuição do custo do aço para a empresa, caso este uso seja correto. Do contrário, os prejuízos gerados podem ter um maior impacto econômico.

Devido à importância do comportamento do fluxo de gás com o líquido na panela, esse assunto é correntemente estudado por vários pesquisadores como Iguchi et. al. [6], que utilizaram uma sonda eletrorresistiva para investigar os efeitos da variação da vazão de gás, a forma e o tamanho do bocal (nozzle) sobre a fração de gás na panela e a frequência de bolhas da pluma. Com isso, os autores foram capazes de correlacionar várias características importantes para os tratamentos que utilizam a injeção de gás. Além disso, Krishnapisharody e Irons [3] fizeram um levantamento dos detalhes de experimentos de diversos outros pesquisadores, incluindo a dimensão do banho, tipo de injetor de gás, tamanho do injetor e sistema estudado e a partir de seus resultados puderam achar boas correlações entre diversas variáveis. Outro trabalho importante é o de Anagbo e Brimacombe [7], onde os autores mostram o comportamento das bolhas geradas por um plugue poroso, além de mostrarem a importância dos tratamentos com plugue poroso em baixas vazões de gás no regime de pequenas bolhas.

Mesmo havendo diversos estudos sobre o tema, ainda existe muita margem para estudos da dinâmica gás/líquido no banho metálico [8]. Um fator muito importante neste tipo de tratamento é a área superficial gás/líquido. Essa área afeta bastante na cinética das reações de refino e dissolução de ligas. Além disso, a área superficial afetará muito a remoção de inclusões do banho.

Este trabalho surgiu de estudo prévio feito pelos próprios autores [9], onde foi observado o potencial de melhoria na remoção de inclusões através de ajustes no plugue e em sua operação. Além disso, experimentos feitos pelos mesmos autores contrapõem alguns trabalhos na literatura. Assim, o estudo foi feito em modelo físico de uma panela de aciaria com injeção através de plugue poroso e tem o objetivo de avaliar a influência da vazão de gás e da área do superficial do plugue nos tamanhos das bolhas geradas em diferentes alturas e na área superficial total das bolhas.

\section{MATERIAIS E MÉTODOS}




\subsection{Descrição dos Experimentos}

Todos os testes foram feitos utilizando modelamento físico. O fluido que simula o aço é a água e o fluido que simula o gás inerte é o ar atmosférico. Para adequação das condições de operação do modelo físico, foram respeitados critérios de similaridade, conforme indicado nos trabalhos de Irons et. al.[1], Elias [10], Mazumdar e Evans [11] e Mazumdar e Guthrie [12].

Como mencionado anteriormente, os testes foram feitos em um modelo físico de panela de aciaria em escala $1 / 5$ de uma panela com capacidade para 180t de aço. As dimensões do modelo de acrílico são mostradas na figura 1.
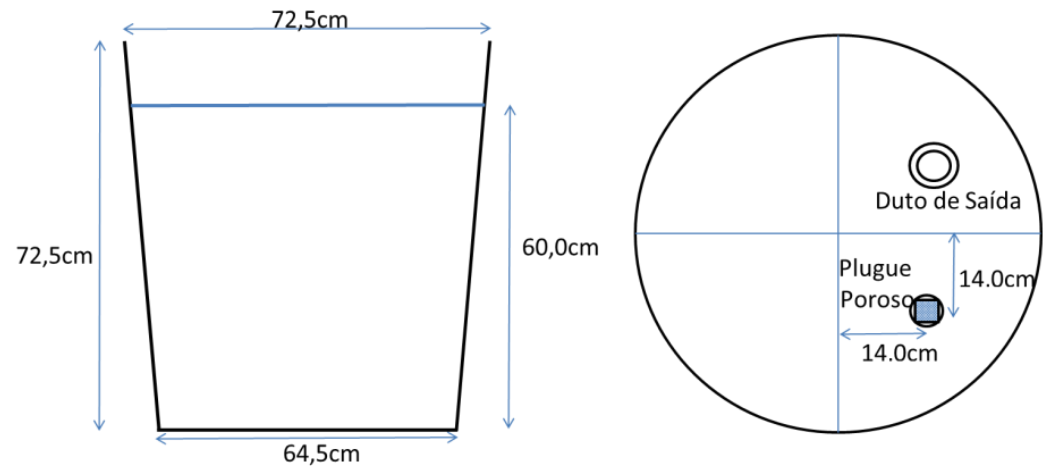

Figura 1 - Dimensões do modelo de panela de aciaria (vista lateral à esquerda e superior à direita)

O modelo apresenta diâmetro inferior de $64,5 \mathrm{~cm}$, diâmetro superior de $72,5 \mathrm{~cm}$, altura total de $72,5 \mathrm{~cm}$ e altura da coluna de água de água de $60 \mathrm{~cm}$. Para a injeção de gás, foi utilizado um compressor e para a regulagem da vazão foi utilizado um rotâmetro. A relação entre a vazão de gás do modelo (laboratório) e vazão do protótipo (indústria) é dada pela equação 1 :

$$
Q_{\text {mod }}\left(\frac{N l}{\min }\right)=0,95 Q_{\text {prot }}\left(\frac{N m^{3}}{h}\right)
$$

As outras relações entre modelo e protótipo são dadas pelas equações 2 e 3 :

$$
\begin{aligned}
& A_{\text {mod }}\left(m^{2}\right)=A_{\text {prot }} \lambda^{2} \\
& H_{\text {mod }}=H_{\text {prot }} \lambda
\end{aligned}
$$

Onde $Q_{\text {mod }}$ é a vazão no modelo, Qprot é a vazão no protótipo, $A_{\bmod }$ é a área no modelo, Aprot é a área no protótipo, $\lambda$ é o fator de escala $(0,2), H_{\text {mod }}$ é a altura no modelo e Hprot é a altura no protótipo.

O número adimensional utilizado como critério de similaridade foi o número de Froude $(\mathrm{Fr})$, que é um balanço entre forças de inércia e forças gravitacionais. $\mathrm{O}$ número de Froude do modelo e do protótipo é o mesmo. Este número é dado pela relação da equação 4:

$$
F r=\frac{F_{I}}{F_{G}}=\frac{U^{2}}{g L}
$$

Onde $F_{I}$ representa as forças de inércia, $F_{G}$ representa as forças gravitacionais, $U$ é a velocidade do líquido, $g$ é a aceleração da gravidade e $L$ é uma dimensão característica.

Foram avaliados os efeitos de diversas variáveis, como: área superficial do plugue, vazão de gás e altura das bolhas. Os valores empregados foram:

- Área Superficial dos plugues no modelo físico:

$$
\begin{array}{ll}
\circ & 15,0 \times 10^{-4} \mathrm{~m}^{2} \text { (Plugue 1) } \\
\circ & 8,6 \times 10^{-4} \mathrm{~m}^{2} \text { (Plugue 2) } \\
\circ & 3,6 \times 10^{-4} \mathrm{~m}^{2} \text { (Plugue 3) }
\end{array}
$$

- Vazões de gás no modelo: 1 a $10 \mathrm{Nl} / \mathrm{min}$; 
- Alturas na panela onde foram analisados os diâmetros das bolhas: $0,1,0,3$ e $0,5 \mathrm{~m}$.

A figura 2 mostra uma foto dos três plugues utilizados para injeção de gás. Estes plugues foram confeccionados utilizando o mesmo material empregado nos plugues industriais.

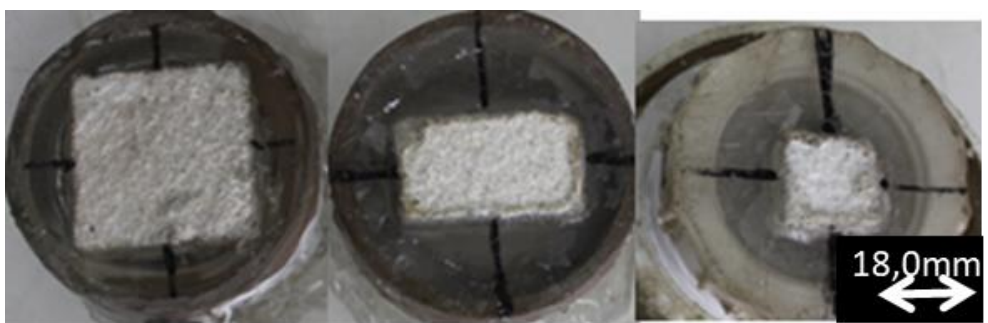

Figura 2 - Plugues utilizados para injeção de gás (plugue 1, plugue 2 e plugue 3 da esquerda para direita)

\subsection{Obtenção das Imagens}

Para obtenção das imagens, a panela foi enchida até a altura de água (mostrada na figura 1) para simular a altura real de aço na panela. $O$ plugue poroso foi posicionado no fundo da panela na mesma posição do plugue real de aciaria (como mostrado na figura 1).

A captação das imagens foi feita utilizando uma câmera Canon EOS 60D com fotos em alta velocidade e em alta resolução.

Foram tomadas diversas fotos da panela em cada uma das condições estudadas e assim foram escolhidas três fotos em cada condição. As fotos foram selecionadas considerando a qualidade de imagem obtida. Assim, os tratamentos e análises puderam ser feitos com maior precisão e nitidez. A montagem dos experimentos é mostrada na figura 3.

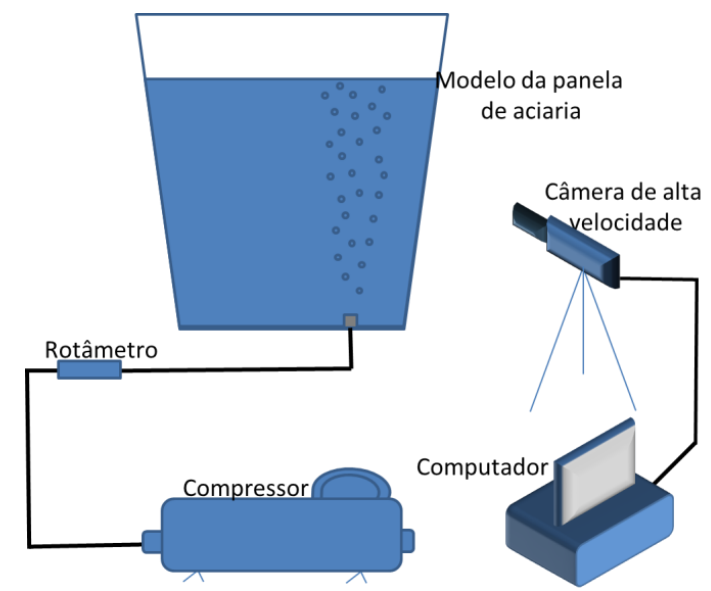

Figura 3 - Montagem do experimento

\subsection{Tratamento das Imagens}

Foi utilizado o software ImageJ para medição dos tamanhos das bolhas. Este software utiliza uma escala conhecida como referência e a partir da quantidade de pixels, ele determina outras dimensões nesta imagem.

Em cada foto, foram avaliadas 30 bolhas, totalizando 90 bolhas para cada condição. As bolhas foram medidas em dois eixos: vertical (a) e horizontal (b). Considerou-se que seu terceiro eixo tinha a mesma dimensão do eixo horizontal. Essa consideração é feita porque as forças atuantes nestes dois eixos são semelhantes dentro da panela.

Dessa forma, o volume de cada um desses elipsoides é dado pela equação 5: 


$$
V=\frac{4}{24} \pi a b^{2}
$$

$\mathrm{Na}$ análise foi também empregado o conceito de diâmetro equivalente. Por este conceito, o diâmetro de cada bolha é o diâmetro equivalente a uma esfera de mesmo volume que o elipsoide medido. Desta forma, o diâmetro (D) de cada esfera igual ao expresso na equação 6:

$$
D=\sqrt[3]{a b^{2}}
$$

Para a medição da área superficial das bolhas foram tomadas fotos da panela inteira, em que a pluma aparece por completo. Essas fotos foram tratadas com o software ImageJ. Esse tratamento visa ressaltar cada bolha e fornecer a área que cada uma das bolhas ocupa na imagem a partir de uma referência fornecida.

A figura 4 mostra a foto original e o tratamento feito pelo ImageJ.

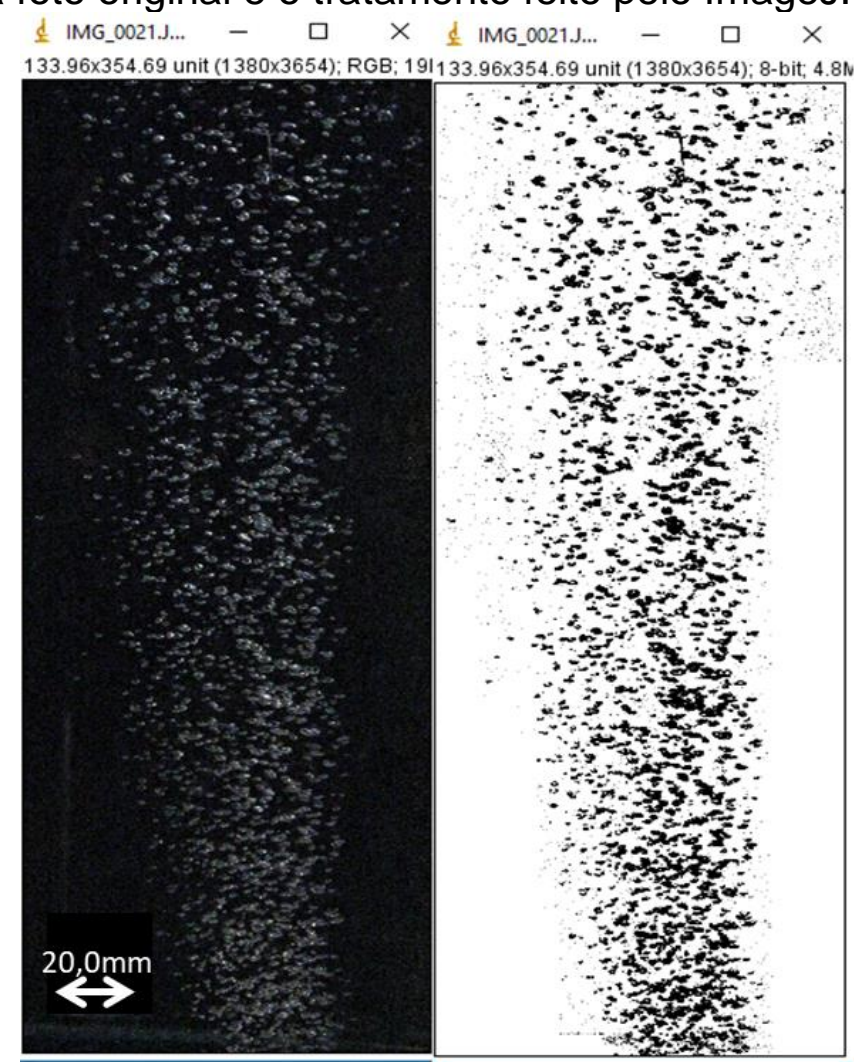

Figura 4 - Foto da pluma à esquerda e tratamento feito pelo software ImageJ à direita

Para a avaliação da área superficial de cada bolha, foi feita a consideração de que elas são esféricas. Sendo assim, a relação de área da bolha na figura e área superficial é dada pela equação 7:

$$
A_{\text {sup }, \text { bolha }}=A_{\text {foto,bolha }} \cdot 4
$$

Essa relação vem das relações de área do círculo (foto) e área superficial de uma esfera.

Os resultados obtidos são apresentados e discutidos a seguir.

\section{RESULTADOS E DISCUSSÃO}

A tabela 1 apresenta um sumário dos resultados obtidos para os diâmetros e áreas superficiais das bolhas.

Tabela 1 - Resultados encontrados para diâmetro médio das bolhas e área superficial em todas as condições

\begin{tabular}{|c|c|c|c|c|}
\hline $\begin{array}{c}\text { Área Superficial do } \\
\text { Plugue }\left(\mathbf{m}^{2}\right)\end{array}$ & Vazão (NI/min) & Altura (m) & $\begin{array}{c}\text { Diâmetro médio das } \\
\text { bolhas }(\mathbf{m m})\end{array}$ & $\begin{array}{c}\text { Área superficial } \\
\text { Total } \mathbf{x} \mathbf{1 0}^{3} \\
\left(\mathbf{m m}^{2}\right)\end{array}$ \\
\hline Plugue 1: $15,0 \times 10^{-4}$ & 1,0 & 0,1 & 1,9 & 29 \\
\cline { 3 - 4 }
\end{tabular}




\begin{tabular}{|c|c|c|c|c|}
\hline & & 0,5 & 2,4 & \\
\hline & \multirow{3}{*}{2,0} & 0,1 & 1,8 & \multirow{3}{*}{41} \\
\hline & & 0,3 & 2,2 & \\
\hline & & 0,5 & 2,9 & \\
\hline & \multirow{3}{*}{4,0} & 0,1 & 2,2 & \multirow{3}{*}{63} \\
\hline & & 0,3 & 2,3 & \\
\hline & & 0,5 & 2,9 & \\
\hline & \multirow{3}{*}{6,0} & 0,1 & 3,7 & \multirow{3}{*}{67} \\
\hline & & 0,3 & 3,8 & \\
\hline & & 0,5 & 3,7 & \\
\hline & \multirow{3}{*}{8,0} & 0,1 & 2,3 & \multirow{3}{*}{74} \\
\hline & & 0,3 & 3,2 & \\
\hline & & 0,5 & 3,6 & \\
\hline & \multirow{3}{*}{10,0} & 0,1 & 3,5 & \multirow{3}{*}{78} \\
\hline & & 0,3 & 3,8 & \\
\hline & & 0,5 & 3,7 & \\
\hline \multirow{18}{*}{ Plugue 2: $8,6 \times 10^{-4}$} & \multirow{3}{*}{1,0} & 0,1 & 2,3 & \multirow{3}{*}{34} \\
\hline & & 0,3 & 2,9 & \\
\hline & & 0,5 & 2,7 & \\
\hline & \multirow{3}{*}{2,0} & 0,1 & 3,2 & \multirow{3}{*}{43} \\
\hline & & 0,3 & 3,4 & \\
\hline & & 0,5 & 3,2 & \\
\hline & \multirow{3}{*}{4,0} & 0,1 & 2,9 & \multirow{3}{*}{57} \\
\hline & & 0,3 & 3,6 & \\
\hline & & 0,5 & 3,9 & \\
\hline & \multirow{3}{*}{6,0} & 0,1 & 3,8 & \multirow{3}{*}{60} \\
\hline & & 0,3 & 4,3 & \\
\hline & & 0,5 & 4,9 & \\
\hline & \multirow{3}{*}{8,0} & 0,1 & 3,2 & \multirow{3}{*}{57} \\
\hline & & 0,3 & 4,4 & \\
\hline & & 0,5 & 4,6 & \\
\hline & & 0,1 & 4,6 & \\
\hline & 10,0 & 0,3 & 4,3 & 59 \\
\hline & & 0,5 & 4,0 & \\
\hline & & 0,1 & 2,6 & \\
\hline & 1,0 & 0,3 & 3,1 & 27 \\
\hline & & 0,5 & 2,8 & \\
\hline & & 0,1 & 3,3 & \\
\hline & 2,0 & 0,3 & 3,4 & 32 \\
\hline & & 0,5 & 3,6 & \\
\hline & & 0,1 & 3,4 & \\
\hline & 4,0 & 0,3 & 3,5 & 27 \\
\hline Pluaue $3 \cdot 3.6 \times 10^{-4}$ & & 0,5 & 3,7 & \\
\hline Plugue $3: 3,6 \times 10^{-4}$ & & 0,1 & 3,6 & \\
\hline & 6,0 & 0,3 & 4,5 & 27 \\
\hline & & 0,5 & 5,4 & \\
\hline & & 0,1 & 4,3 & \\
\hline & 8,0 & 0,3 & 5,2 & 26 \\
\hline & & 0,5 & 4,3 & \\
\hline & & 0,1 & 5,3 & \\
\hline & 10,0 & 0,3 & 4,6 & 31 \\
\hline & & 0,5 & 5,0 & \\
\hline
\end{tabular}

Com base nos dados da tabela acima, foram criados gráficos para análise dos resultados.

Para análise do tamanho das bolhas, foram feitos os gráficos das figuras 5, 6 e 7. A figura 5 mostra o diâmetro das bolhas em relação à altura delas na panela. 


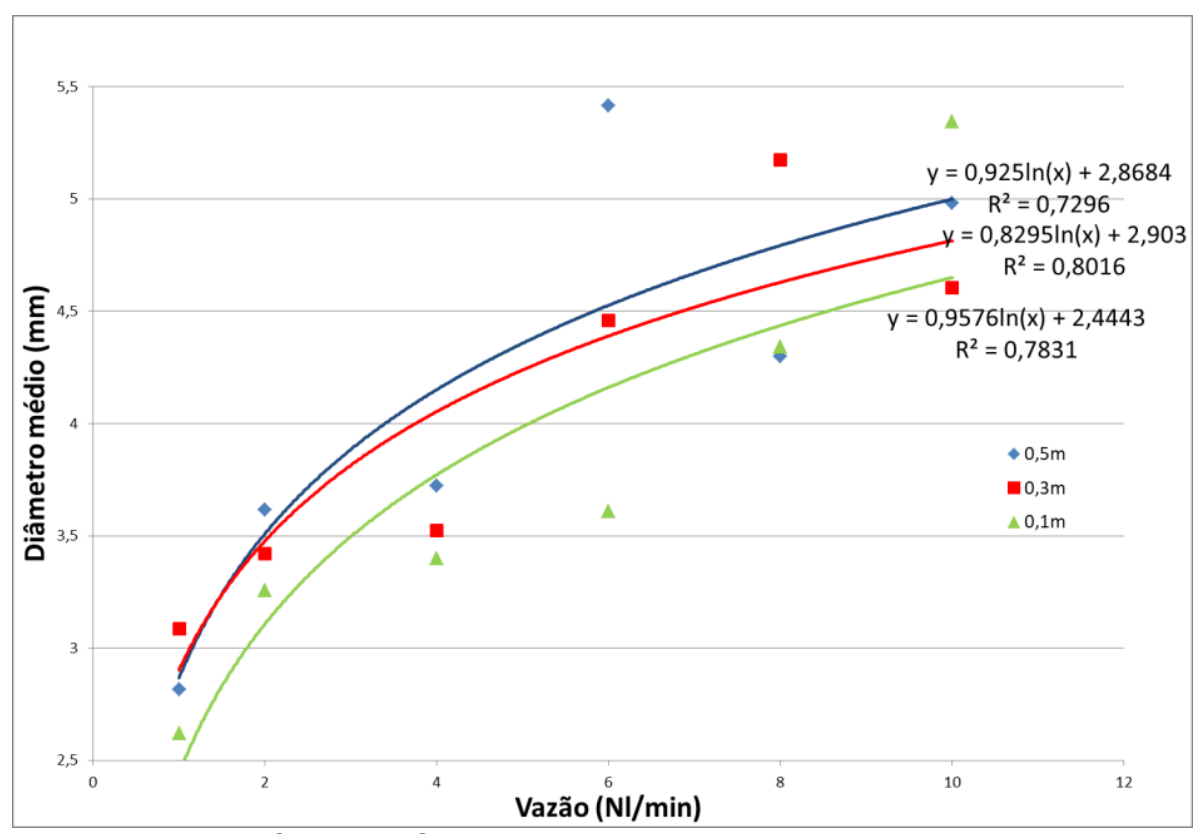

Figura 5 - Diâmetro médio das bolhas em cada altura para o plugue 3

Analogamente ao gráfico da figura 5 , feita para o plugue 3 , também foram feitas análises para os outros plugues.

O melhor ajuste para os pontos encontrados foi o ajuste logarítmico. Nota-se que há uma tendência de aumento do diâmetro das bolhas com o aumento da vazão e também uma tendência do aumento do diâmetro das bolhas com o aumento da altura da coluna de bolhas.

A figura 6 mostra as médias dos diâmetros das bolhas nas alturas avaliadas em relação à vazão para os três plugues:

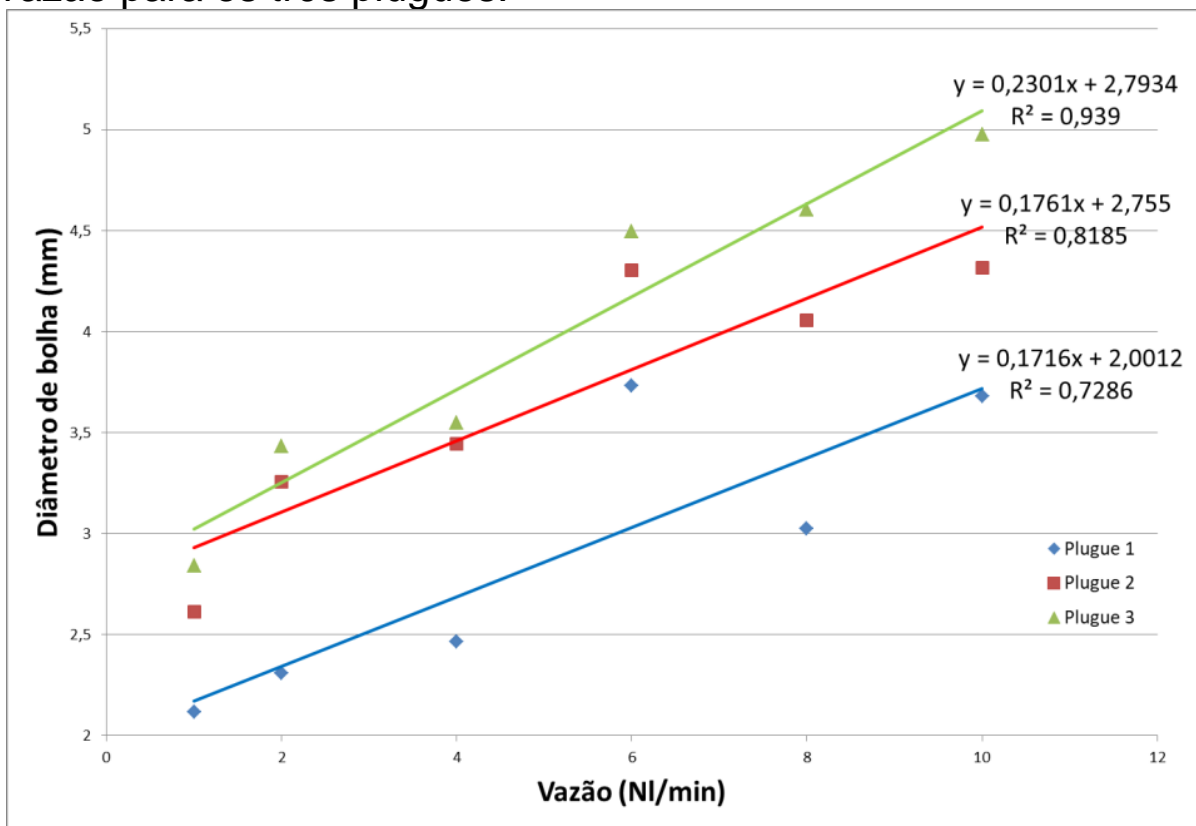

Figura 6 - Comparação de diâmetro médio das bolhas entre os 3 plugues

Para o gráfico da figura 6, o melhor ajuste foi linear. Como mostrado anteriormente e ressaltado nesta figura, existe uma tendência do aumento do tamanho das bolhas com o aumento da vazão. Existe também a tendência do aumento do tamanho das bolhas com a diminuição da área superficial do plugue. A figura 7 mostra o gráfico 
do tamanho de bolha em relação à vazão de gás dividida pela área superficial do plugue:

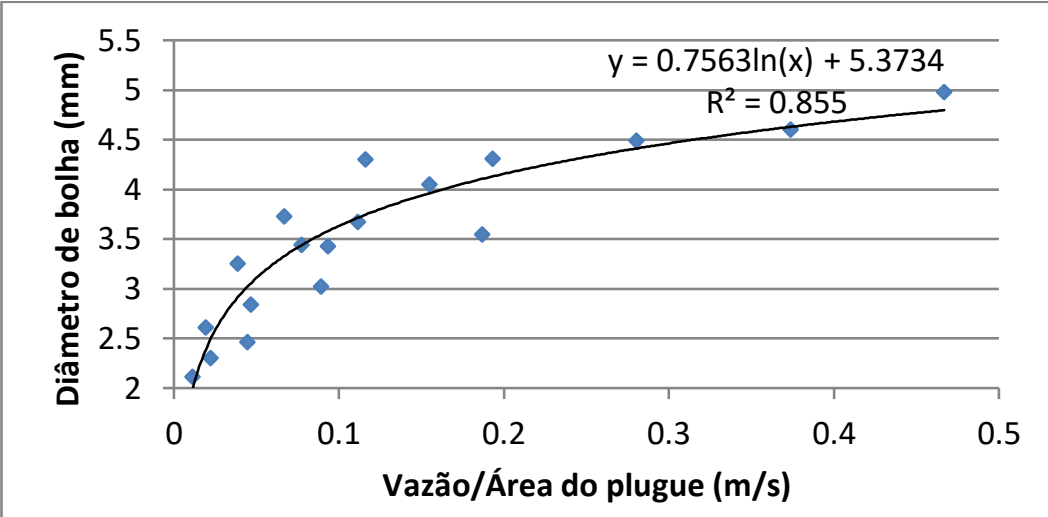

Figura 7 - Diâmetro das bolhas em relação à vazão por área do plugue $(\mathrm{m} / \mathrm{s})$

Quando analisado em termos da razão vazão de gás pela área superficial do plugue, o diâmetro das bolhas apresenta um ajuste muito bom. Isso mostra que esse diâmetro das bolhas não depende apenas da vazão, mas também da área superficial dos plugues.

Porém, quando se fala em tratamento do aço, o conhecimento do diâmetro das bolhas não é suficiente para um bom tratamento. É necessário o conhecimento da área superficial gerada pelas bolhas, pois a cinética das reações e a captura das inclusões dependem dessa área.

Para o estudo da área superficial das bolhas foram feitos os gráficos das figuras 8, 9 e 10. A figura 8 apresenta o gráfico da área superficial das bolhas para o plugue 1 .

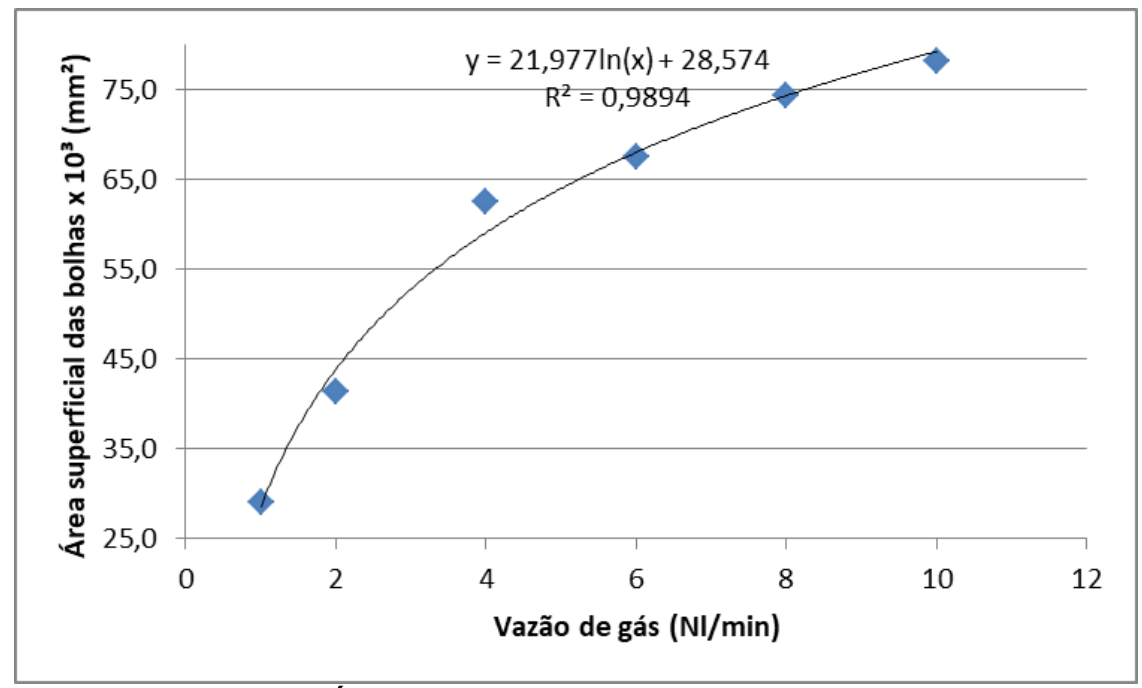

Figura 8 - Área superficial das bolhas para o plugue 1

Nesta imagem foi identificado que o melhor ajuste para os dados analisados é o ajuste logarítmico. Observa-se uma relação crescente entre a vazão de gás e área superficial.

A figura 9 apresenta o gráfico para o plugue 2 . 


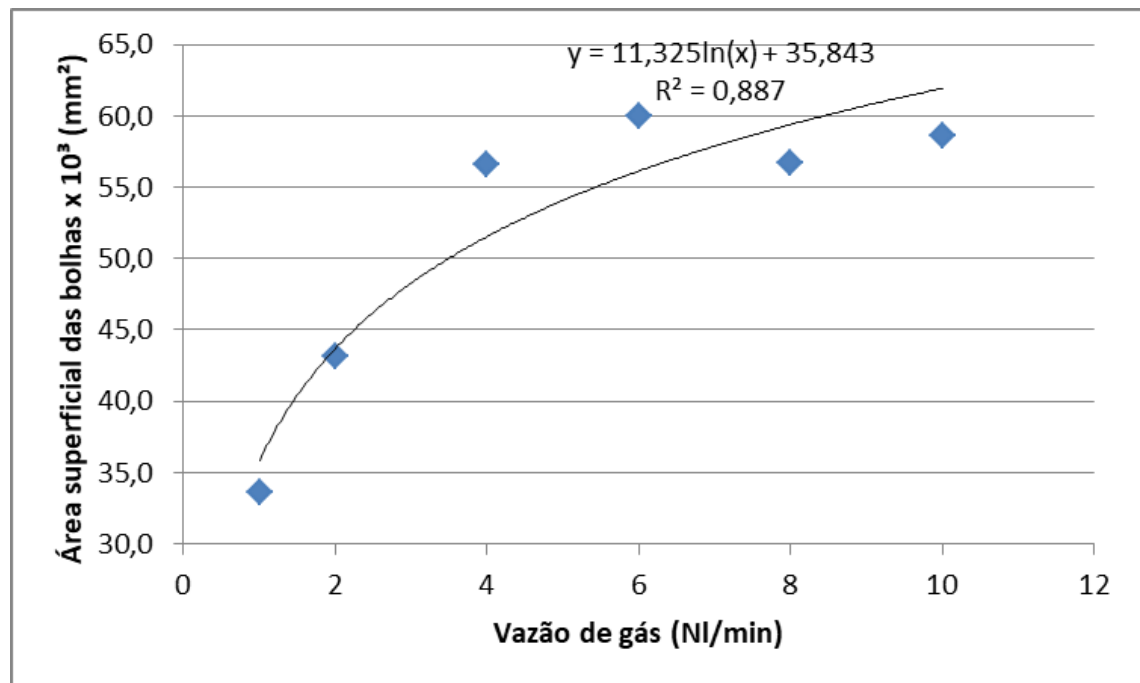

Figura 9 - Área superficial das bolhas para o plugue 2

Já na figura 9 a regressão logarítmica não apresenta um ajuste tão bom quando comparado à curva do plugue 1. Isso ocorre pela presença de coalescência das bolhas nas vazões mais altas.

A figura 10 apresenta o gráfico para o plugue 3.

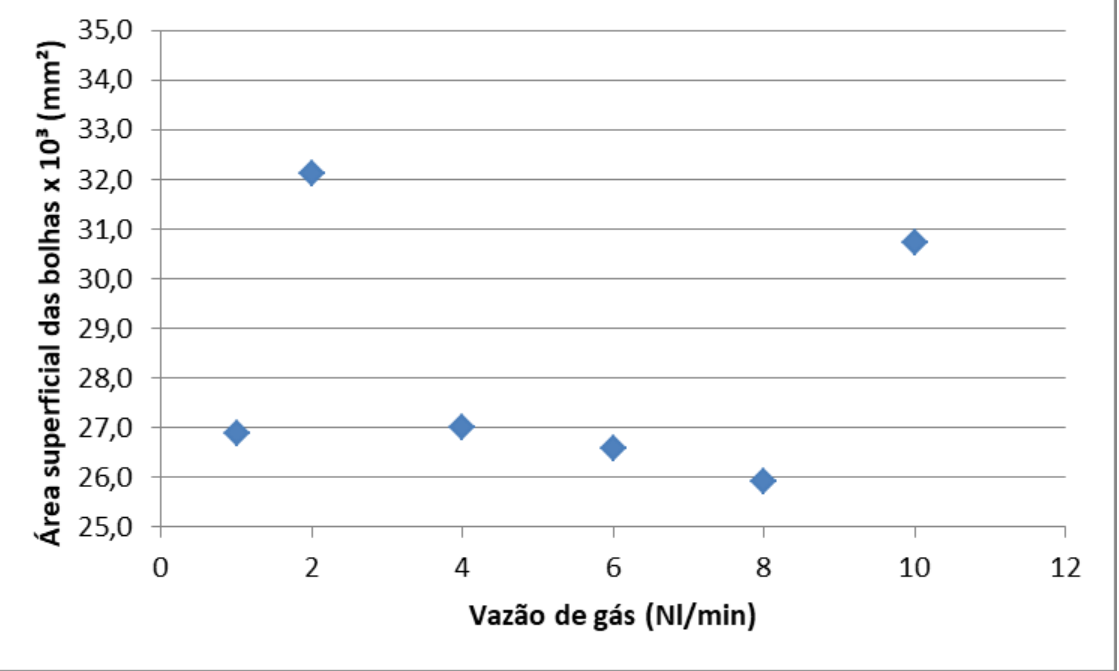

Figura 10 - Área superficial das bolhas para o plugue 3

Não foi possível achar uma relação direta entre a vazão e a área superficial das bolhas para o plugue 3. O que pode ser justificado pelo alto grau de coalescimento das bolhas, como ilustrado na figura 11, que mostra as fotos das plumas formadas pelo plugue 3 para cada vazão. Nesta figura são observados os diferentes estágios do borbulhamento de gás. Nas duas primeiras vazões, pode-se constatar uma distribuição de bolhas pequenas, nas duas vazões intermediarias (4 e $6 \mathrm{NI} / \mathrm{min}$ ) observa-se um regime transiente de bolhas pequenas e grandes. Nas vazões 8 e $10 \mathrm{NI} / \mathrm{min}$ são identificadas praticamente apenas a formação de bolhas grandes, decorrentes da maior coalescência. 


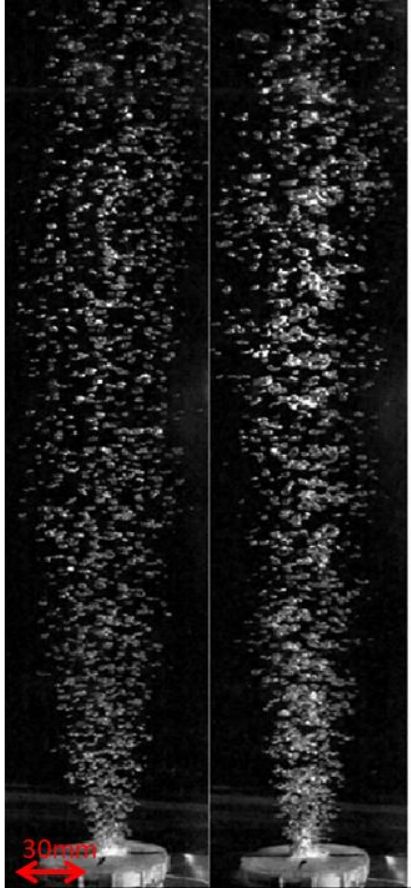

$1 \mathrm{Nl} / \mathrm{min}$

$2 \mathrm{NI} / \mathrm{min}$

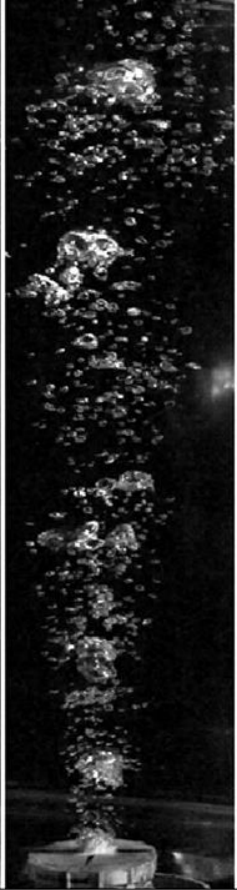

$4 \mathrm{NI} / \mathrm{min}$

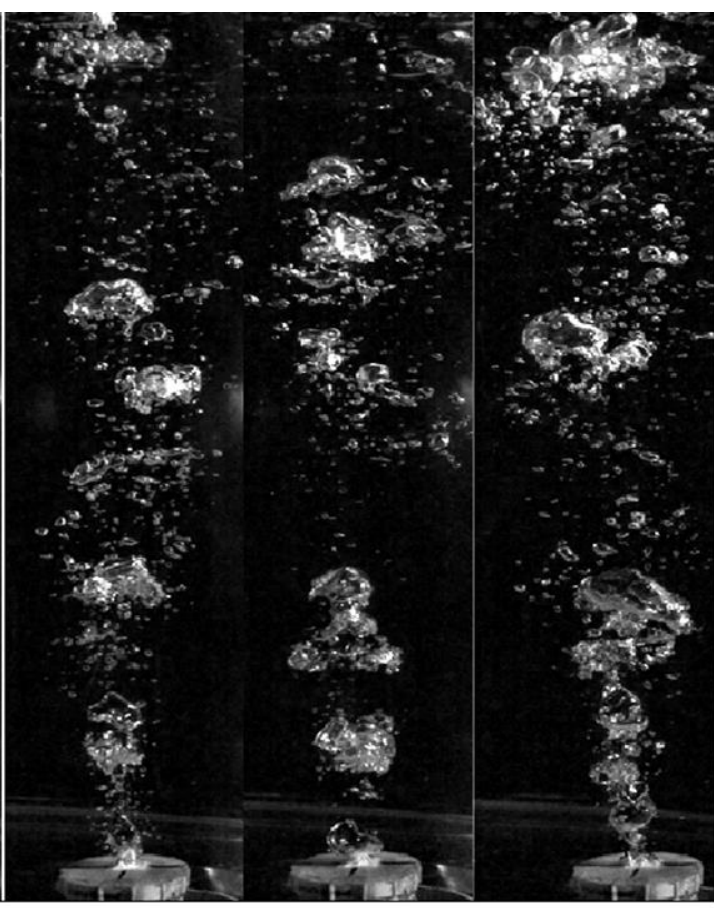

$6 \mathrm{NI} / \mathrm{min}$

$8 \mathrm{NI} / \mathrm{min}$

$10 \mathrm{NI} / \mathrm{min}$

Figura 41 - Perfis de dispersão de bolhas de um plugue poroso

Embora essa relação de área superficial das bolhas em função da vazão de gás não possa ser descrita como uma curva logarítmica, pode-se observar que no momento em que não ocorre coalescimento das bolhas, a área superficial aumenta (vazões de $1 \mathrm{Nl} / \mathrm{min}$ e $2 \mathrm{NI} / \mathrm{min}$ ). Já quando acontece o inicio do coalescimento ou regime de coalescimento transiente, a área superficial das bolhas diminui em relação às vazões anteriores e tende a permanecer constante enquanto durar este regime. No momento em que a coalescência está estabelecida com grandes bolhas (vazões de $8 \mathrm{Nl} / \mathrm{min}$ e $10 \mathrm{Nl} / \mathrm{min}$ ), a área superficial das bolhas volta a aumentar.

Quando comparada a duas bolhas pequenas, uma bolha maior (de mesmo volume que a soma dessas duas) tem área superficial menor. Esse é o motivo da coalescência ter um efeito na diminuição da área superficial.

A partir do momento em que a coalescência está estabelecida e as grandes bolhas são formadas na base do plugue, com o aumento da vazão, estas bolhas tendem a aumentar seu diâmetro. Isso faz com que a área superficial também volte a aumentar, como mostrado no final do gráfico.

O gráfico da figura 12 mostra a variação da área superficial com o aumento da vazão para cada plugue. Os valores entre 1 e $2 \mathrm{Nl} / \mathrm{min}$ do plugue 1 e plugue 2 estão diferentes da tendência geral. Por isso foi feito um teste t para duas amostras usando um software estatístico para a certeza da diferença desses valores.

Para o teste t para duas amostras comparando a vazão de $1 \mathrm{NI} / \mathrm{min}$ entre o plugue 1 e 2, o valor tobs $=-12,39$ pertence à região crítica $(t<-1,96$ ou $t>1,96)$ e além disso, o valor-p achado foi de 0,0 , sendo menor que a significância do teste $\alpha=0,05$. Essas duas constatações fazem com que a hipótese de médias iguais seja rejeitada. Realizando o teste t para duas amostras novamente, agora para a vazão de $2 \mathrm{~N} / \mathrm{min}$ entre o plugue 1 e o plugue 2 , o valor tobs $=-4,54$, que pertence à região crítica $(t<$ $-1,96$ ou $t>1,96)$ e o valor-p achado foi de 0,0 , sendo menor que o nível de significância do teste $\alpha=0,05$. Essas duas constatações fazem com que a hipótese de que as médias sejam iguais seja rejeitada. 


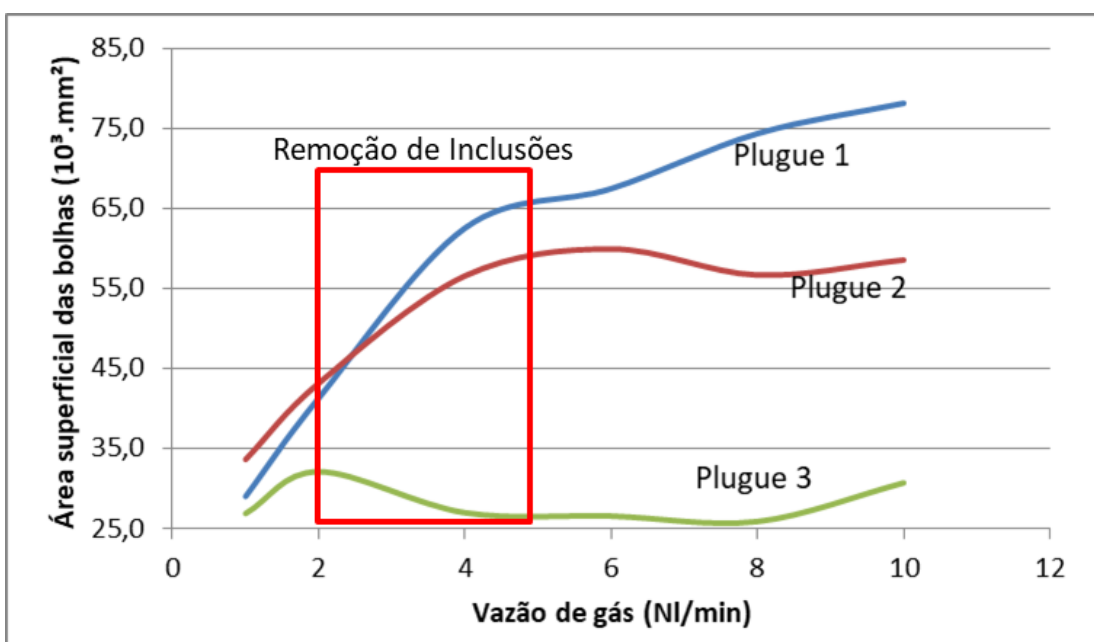

Figura 12 - Aumento da área superficial com o aumento da vazão para todos os plugues

Sendo assim, pode-se concluir que os valores médios para as amostras tanto para as vazões de $1 \mathrm{~N} / /$ min quanto para de $2 \mathrm{~N} / /$ min são diferentes com $95 \%$ de confiança. Na prática industrial para remoção de inclusões, as vazões utilizadas são baixas, por volta de 2 a $5 \mathrm{Nm}^{3} / \mathrm{h}$. Este valor leva em conta a não abertura da camada de escória e a não projeção de aço para fora da panela. Este valor está representado no gráfico da figura 12, já convertido para os valores laboratoriais. Sendo assim, o plugue que fornece maior área superficial de bolhas será o maior plugue (plugue 1).

Outros tratamentos em que se pode abrir a camada de escória, onde são empregados valores maiores de vazão de gás (acima de $10 \mathrm{Nm}^{3} / \mathrm{h}$ ) também deve-se utilizar um plugue de maior área superficial. Os estudos mostram que a coalescência das bolhas aumenta seu diâmetro médio, porém a diminuição no número total de bolhas faz com que a área superficial seja menor.

Finalmente, é interessante comparar os resultados do presente trabalho com o de outros trabalhos anteriores. Quanto ao tamanho das bolhas, diversos outros trabalhos fizeram essa medição para os tratamentos na panela. A comparação entre os trabalhos se torna complicada uma vez que as condições dos experimentos são bem diferentes, como área superficial do plugue, fluidos usados, vazão de gás, altura do banho, tipo de injetor e outros. Anagbo e Brimacombe [7] fizeram experimentos utilizando plugue poroso e o resultado para os diâmetros médios de bolha foi muito maior do que os diâmetros médios encontrados neste trabalho, chegando a $50 \mathrm{~mm}$. Porém, a vazão utilizada foi muito superior e a área do plugue também. Outros autores, como Castello-Branco [8] e Okumura [13] também fizeram essas medidas utilizando ventaneira e os valores encontrados também foram maiores, chegando a $100 \mathrm{~mm}$. O que já era esperado pelo tipo de injetor usado. Outro experimento com análise do tamanho de bolhas acontece em processos que utilizam injeção de gás na válvula longa (válvula que liga a panela e o distribuidor para não haver contato do aço com ar atmosférico). As bolhas criadas têm tamanho reduzido devido a alta turbulência na região. Wang et. al. [14] mostram que em altas vazões de gás, as bolhas são geradas com tamanhos menores que $0,5 \mathrm{~mm}$. Já em 2016, Chang et al. [15] conseguiram através da mesma técnica chegar a bolhas de $0,5 \mathrm{~mm}$ porém em seus experimentos, o aumento da vazão de gás faz com que o tamanho das bolhas aumente.

\section{CONCLUSÃO}

A variação da vazão afeta significativamente o diâmetro das bolhas e a sua área superficial total. Quanto maior a vazão, maior será o diâmetro das bolhas, seguindo 
uma tendência logarítmica quando comparados em uma mesma altura ao longo da panela. Já quando se compara a média dos tamanhos de bolhas em todas as alturas, essa tendência é linear.

Embora o tamanho de bolha seja de grande importância para a área superficial, outros fatores como a quantidade de bolhas geradas também é muito importante. Por isso, apesar de apresentar os menores valores para tamanho de bolhas, 0 plugue 1 tem maior área superficial total de bolhas, para a maioria das vazões, pois as gera em maior quantidade.

Nota-se que o plugue 2 em baixas vazões apresenta maior área superficial total de bolhas. Esses valores foram testados estatisticamente e foram aceitos com $95 \%$ de confiança. A razão disso ainda é desconhecida e mais estudos precisam ser feitos para uma conclusão final.

Portanto, para a otimização dos tratamentos do refino secundário, que visam maior área superficial das bolhas, um plugue com maior área e vazões maiores são recomendadas, fora do regime de coalescimento e levando em consideração a possibilidade de abertura do olho. Outros testes ainda precisam ser feitos para avaliar a abertura do olho nas vazões e plugues estudados.

\section{Agradecimentos}

Os autores agradecem ao Programa de Pós Graduação em Engenharia Metalúrgica, de Minas e de Materiais (PPGEM) da UFMG, aos programas de fomento à pesquisa CAPES-PROEX, CNPq e FAPEMIG pelo apoio ao projeto.

\section{REFERÊNCIAS}

[1] Irons G, Senguttuvan A, Krishnapisharody K. Recent advances in fluid dynamics of ladle metallurgy. ISIJ International. 2015;55(1): 1-6;

[2] Irons GA, Krishnapisharody K. A unified approach to the fluid dynamics of gas-liquid plumes in ladle metallurgy. ISIJ International. 2010;50(10): 1413-1421;

[3] Krishnapisharody K, Irons GA. A critical review of the modified froude number in ladle metallurgy. Metallurgical and Materials Transactions B. 2013; 44B: 1486-1498;

[4] Krishnapisharody K, Irons GA. An analysis of recirculatory flows in gas-stirred ladles. Steel Research Inst. 2010; 81(10): 880-885;

[5] Krishnapisharody K, Irons GA. An extended model for slag eye size in ladle metallurgy. ISIJ International. 2008; 48(12): 1807-1809;

[6] Iguchi M, Demoto Y, Sugawara N, Morita Z. Bubble behavior in $\mathrm{Hg}$-air vertical bubbling jets in a cylindrical vessel. ISIJ International. 1992; 32 (9): 998-1005;

[7] Anagbo PE, Brimacombe JK. Plume characteristics and liquid circulation in gas injection through a porous plug. Metallurgical Transactions B. 1990; 21 (B): 637-648;

[8] Castello-Branco MASC, Schwerdtfeger K. Large-scale measurements of the physical characteristics of round vertical bubble plumes in liquids. Metallurgical and Materials Transactions B. 1994; 25B: 359-371;

[9] Silva PHR, Cardoso TTM, Mendonça AFG, Nascimento AA, Tavares RP. Estudo da remoção de inclusões por injeção de gás inerte na panela por modelamento físico. Anais $46 \circ \mathrm{Sem}$. Aciaria, ABM Week. 2015;

[10] Elias RCA. Modelamento Físico e Matemático da Placa de Mistura no Lingotamento Contínuo Efeitos de Modificadores de Fluxo no Molde. Dissertação de Mestrado. PPGEM, UFMG. 2010;

[11] Mazumdar D, Evans JW. Modeling of steelmaking processes. Boca Raton: CRC Press; 2010;

[12] Mazumdar D, Guthrie RIL. The physical and mathematical modelling of gas stirred ladle systems. ISIJ International. 1995; 35(1):1-20;

[13] Okumura K, Komarov SV, Sano M. Gas injection from slot nozzle with various shapes in water.

ISIJ International, 2000; 40(6): 544-548;

[14] Wang L, Lee HG, Hayes P. A new approach to molten steel refining using fine bubbles. ISIJ International. 1996; 36 (1): 17-24;

[15] Chang S, Cao X, Hsin CH, Zou Z, Isac M, Guthrie RIL. Removal of inclusion using micro-bubble swarms in a four-strand, full-scale, water model tundish. ISI International, 2016; 56(7): 1188-1197 